\title{
Rosiglitazone: a European regulatory perspective
}

\author{
E. Blind $\cdot$ K. Dunder $\cdot$ P. A. de Graeff $\cdot$ E. Abadie
}

Received: 18 October 2010 /Accepted: 22 October 2010 /Published online: 14 December 2010

(C) Springer-Verlag 2010

Keywords Cardiovascular outcome studies · Cardiovascular risk · Diabetes mellitus · Drug safety European Medicines Agency $\cdot$ Pioglitazone $\cdot$ RECORD trial $\cdot$ Regulatory approval $\cdot$ Rosiglitazone $\cdot$ Thiazolidinedione

\section{Abbreviations}

$\begin{array}{ll}\text { CHF } & \text { Congestive heart failure } \\ \text { CHMP } & \text { Committee for Medicinal Products for Human } \\ & \text { Use }\end{array}$

The views expressed in this article are the personal views of the authors and should not be understood or quoted as being made on behalf of or reflecting the position of the European Medicines Agency or any of its committees or working parties.

E. Blind $(\bowtie)$

European Medicines Agency (EMA),

7 Westferry Circus, Canary Wharf,

London E14 4HB, UK

e-mail: eberhard.blind@ema.europa.eu

E. Blind

Department of Medicine I, University of Würzburg,

Würzburg, Germany

K. Dunder

Läkemedelsverket (the Medical Products Agency),

Uppsala, Sweden

P. A. de Graeff

Dutch Medicines Evaluation Board,

The Hague, the Netherlands

P. A. de Graeff

Department of Clinical Pharmacology, University of Groningen,

Groningen, the Netherlands

\section{E. Abadie}

Agence Française de Sécurité Sanitaire des Produits de Santé,

Saint-Denis, France

$\begin{array}{ll}\text { EMA } & \text { European Medicines Agency } \\ \text { FDA } & \begin{array}{l}\text { Food and Drug Administration } \\ \text { Marketing authorisation holder }\end{array} \\ \text { PROactive } & \begin{array}{l}\text { Prospective Pioglitazone Clinical Trial in } \\ \text { Macrovascular Events }\end{array} \\ \text { RECORD } & \begin{array}{l}\text { Rosiglitazone Evaluated for Cardiovascular } \\ \text { Outcomes in Oral Agent Combination Thera- } \\ \text { py for Type 2 Diabetes }\end{array} \\ \text { SAG } & \begin{array}{l}\text { Scientific Advisory Group of the EMA } \\ \text { Summary of Product Characteristics }\end{array} \\ \text { TZD } & \begin{array}{l}\text { Thiazolidinedione } \\ \end{array}\end{array}$

\section{Introduction}

Rosiglitazone, a drug for the treatment of type 2 diabetes, which has been authorised for use in the EU since 11 July 2000, has attracted continuous scrutiny with regard to its cardiovascular safety profile. The European Medicines Agency (EMA)'s scientific committee, the Committee for Medicinal Products for Human Use (CHMP), in its most recent comprehensive review of rosiglitazone (started 8 July 2010 and completed 22 September 2010), concluded that the benefits of rosiglitazone no longer outweigh the risks, and that the marketing authorisation of the drug in the EU should be suspended [1].

The concerns about the cardiovascular safety profile of rosiglitazone have stoked an intense debate, not only about the drug itself but also about the regulatory environment of approval and ongoing oversight of diabetes drugs in general. The authors would therefore like to shed some light on key issues of the debate, including questions raised recently in the British Medical Journal [2] and elsewhere in the medical community. 


\section{Initial authorisation of rosiglitazone in $\mathbf{2 0 0 0}$}

Avandia (rosiglitazone) was developed for the treatment of type 2 diabetes and belongs to the thiazolidinedione (TZD) class of drugs. The only other drug in this class to be currently authorised in the EU is Actos (pioglitazone). The mechanism of action of rosiglitazone is (predominantly) as a peroxisome proliferator-activated receptor- $\gamma$ (PPAR $\gamma$ ) receptor modulator. Besides a sustained glucose-lowering effect, TZDs share some pharmacological features, such as an insulin-sensitising effect, which were considered at the time of approval to be of potential additional clinical benefit. Furthermore, at that time, only a limited number of non-insulin glucose-lowering drugs were available as therapeutic options.

One of the safety concerns identified before approval in patients treated with TZDs was fluid retention [3]. Moreover, the combination of rosiglitazone with insulin resulted in a higher rate of congestive heart failure (CHF) compared with insulin alone [3]. A potentially unfavourable effect of rosiglitazone on the lipid profile was also noted. Overall, CHF was clearly recognised as a risk at the time of approval and this was reflected in warnings and contraindications in the product information, the Summary of Product Characteristics (SPC), of Avandia (see Text box). The mechanism underlying the increased risk of CHF was not well understood, being potentially attributable to fluid overload. However, no increase in myocardial infarction or stroke was apparent [3]. From the start, rosiglitazone usage was restricted in the EU to a secondline therapy (see Text box), since an initial request for a monotherapy first-line indication had been rejected by EMA's scientific committee in 1999, mainly due to the uncertain cardiovascular safety profile. Moreover, an outcome study to address the concerns regarding cardiovascular safety was requested by the EMA, and the makers agreed to perform post-marketing a long-term cardiovascular morbidity/mortality study in patients on rosiglitazone in combination with a sulfonylurea or metformin (Rosiglitazone Evaluated for Cardiovascular Outcomes in Oral Agent Combination Therapy for Type 2 Diabetes [RECORD], see below). Subsequent changes to the product information with regard to the cardiovascular profile are summarised in the Text box.

\section{Emerging data of cardiovascular risk associated with the use of rosiglitazone}

Initial reports of a potential increase in the risks of cardiovascular events beyond CHF with the use of rosiglitazone were submitted by the marketing authorisation holder (MAH) to the EMA in 2006, and information on this analysis was included in the product information in September 2006 (see Text box). In 2007, Nissen and Wolski performed a meta-analysis of 42 , mostly small and short-term, trials with rosiglitazone, concluding that there was an increased risk of myocardial infarction and a numerical, but not statistically significant, increase in cardiovascular death when comparing rosiglitazone with placebo or standard diabetes therapy [4]. This study sparked an immediate discussion in the medical community about the benefits and risks of rosiglitazone [5-10]. Similar analyses - largely based on the same set of trials - by the US Food and Drug Administration (FDA) and by the MAH of Avandia gave consistent results. However, none of the trials included in these meta-analyses was designed to generate data on cardiovascular outcome. Furthermore, the RECORD trial data (see below) were not available yet, except for limited data from an interim analysis [11], leaving some uncertainty about the cardiovascular safety profile of rosiglitazone in the absence of fully comprehensive data.

\section{The evidence from cardiovascular outcome trials with TZDs}

To address the concerns about the cardiovascular profile of the drugs, the EU regulators had already in 2000, at time of approval of the TZDs, requested follow-up studies (see above), including long-term cardiovascular outcome studies in each case, now known as the RECORD trial for rosiglitazone [12] and the Prospective Pioglitazone Clinical Trial in Macrovascular Events (PROactive) for pioglitazone [13].

The design of the two trials was different, e.g. addition of a glucose-lowering therapy in PROactive (doubleblind, superiority trial vs placebo) vs an active comparator design in RECORD (open-label, non-inferiority trial vs standard therapy). The populations studied were also different, therefore the two studies cannot be directly compared.

The trial with pioglitazone, published as the PROactive Study in 2005 [13], did show a reduction of $10 \%$ vs placebo in its primary endpoint, a complex composite cardiovascular endpoint, which did not, however, reach statistical significance. Although this study failed in its primary outcome measure, and therefore no claim of cardiovascular outcome benefit was granted in the SPC, it provided reassurance that no increase in the frequency of macrovascular events was associated with its use.

The results of the RECORD trial, however, sparked a controversy that has been extensively discussed elsewhere (for example, see [2]). The results as published [12] show non-inferiority with regard to cardiovascular events and 


\section{Product information for Avandia: indications, contraindications, and SPC label changes concerning the cardiovascular system over time}

March 2000

Positive recommendation for a marketing authorisation of Avandia:

- Use restricted to second-line therapy, as add on to metformin or a sulfonylurea, in patients unsuccessfully treated with either of the two drugs or when metformin is contraindicated

- Contraindicated for heart failure or history of heart failure with regard to all NYHA stages

- Contraindicated for combined use with insulin

- Warning with regard to fluid retention and heart failure

- Restricted to prescription by physicians experienced with treatment of type 2 diabetes

May 2003

Indicated also as second-line monotherapy in patients unsuccessfully treated with metformin or when metformin is contraindicated

November 2004 Indicated also as second-line therapy as add on to metformin and a sulfonylurea in patients unsuccessfully treated with a combination of the two drugs

April 2005 Minor change in the product information, following the review of a study in CHF patients performed by the $\mathrm{MAH}^{\mathrm{b}}$, to inform about the frequency of worsening of heart failure in patients with New York Heart Association stage 1-2

September 2006 Following a review, including a data set of 42 retrospectively analysed trials with rosiglitazone provided by the $\mathrm{MAH}^{\mathrm{c}}$, new information is added:

- Risk of cardiac ischaemia, with a hazard ratio of 1.31 when comparing rosiglitazonecontaining treatments with comparator treatments from this analysis

- Lack of an association of rosiglitazone with a cardiovascular composite endpoint in an observational study

- Additional information on the risk of CHF with rosiglitazone especially in combination with a sulfonylurea or insulin

December $2006^{\mathrm{d}} \quad$ The contraindication of the combined use with insulin is lifted to allow use in exceptional cases and under close supervision only

January 2008 Additional information following a benefit/risk review started in May 2007:

- Contraindicated in acute coronary syndrome

- Warning that treatment with rosiglitazone may be associated with an increased risk of myocardial ischaemic events and its use is therefore not recommended in patients with ischaemic heart disease and/or peripheral arterial disease

- Statement that the available data on the risk of myocardial ischaemia in its entirety is inconclusive

February 2010 Following a review of a dataset of 52 trials provided by the $\mathrm{MAH}^{\mathrm{c}}$ and the RECORD study during 2009, the information in the product information with regard to the risk of myocardial ischaemia has been updated and it is stated that the current data on this remains inconclusive

September 2010 Following the most recent review, recommendation of EMA's CHMP to suspend all marketing authorisations of rosiglitazone-containing drugs with regard to all indications

${ }^{a}$ Only major changes are listed. Dates given are those of the opinion of EMA's Scientific Committee. Fixed-dose combination products containing rosiglitazone (Avandamet and Avaglim) were approved after 2000

${ }^{\mathrm{b}}$ This study was performed by the MAH as a post-approval commitment

${ }^{\mathrm{c}}$ This study was performed by the MAH and some more information will be included in a forthcoming European Public Assessment Report by the EMA

${ }^{\mathrm{d}}$ For Avandamet; followed by implementation for Avandia in October 2007 
cardiovascular death when the treatment with rosiglitazone is compared with metformin or a sulfonylurea. For myocardial infarction, there was a non-statistically significant increase in risk. In their assessment, the European regulators acknowledged weaknesses of the study, such as an unexpectedly low rate of cardiovascular events and the openlabel design, which may lead to reporting bias. They found that the results were inconclusive with regard to cardiovascular events, neither showing definite proof of an increased rate, nor rejecting this hypothesis (this assessment will be included in an upcoming European Public Assessment Report by the EMA). Unsatisfactory as the RECORD trial may appear in some respects, both the PROactive and RECORD trials have been undertaken by the MAHs following the request of $\mathrm{EU}$ regulators a decade ago to perform outcome studies. They represent an important body of data on the cardiovascular profile of TZDs and remain the only sizeable cardiovascular outcome trials on these drugs.

\section{Further developments and new data on rosiglitazone in 2010}

In 2008, the EMA added warnings, including one that treatment with rosiglitazone may be associated with an increased risk of myocardial ischaemic events, and a contraindication to the SPC of Avandia (see Text box). Following the publication of an article by Nissen and Wolski [4], the changes to the product information by the EMA, and the exclusion of rosiglitazone from an ADA/EASD type 2 diabetes treatment algorithm in a paper co-authored by several endocrinologists [14], prescription numbers in the EU declined substantially from 2007 onwards.

Significant new data on the cardiovascular risk of rosiglitazone became available during 2010 . The results of a large epidemiological study by Graham et al. [15], which directly compared the cardiovascular safety of rosiglitazone and pioglitazone, was published on 28 June [15] but leaked earlier to some print media. On the same day, an updated analysis by Nissen and Wolski [16] became available, which confirmed and strengthened the results of their previous meta-analysis [4]. This update, which included the RECORD study, found an increased risk of myocardial infarction but not of cardiovascular death when comparing rosiglitazone with placebo or standard diabetes therapy. The study by Graham et al. [15] suggested that, when directly compared with pioglitazone, rosiglitazone was more frequently associated with cardiovascular events but not myocardial infarction, but its interpretation was made difficult by its observational design. Since new data had become available, the EMA promptly started its most recent review of rosiglitazone. Subsequently, the FDA, in preparing their Advisory Committee Meeting on Avandia on 13 and 14 July 2010, contributed additional analyses, among them two new comprehensive meta-analyses of the cardiovascular outcomes of 52 studies with rosiglitazone and 29 studies with pioglitazone, respectively, showing a trend for reduction of risk for pioglitazone and for an increase for rosiglitazone [17]. Yet, the results of another observational study comparing the cardiovascular safety of rosiglitazone with pioglitazone were published on 25 August 2010 [18], and these did not show a difference in risk. However, the database consisted of a relatively young population with a low risk of cardiovascular events. In August 2010, the EMA conducted a retrospective cohort study to estimate adherence to the restriction in the label of rosiglitazone [19]. The study was based on an analysis of routine coding of clinical events and prescriptions by primary care doctors involving about four million patients in the UK from the THIN (The Health Improvement Network) database between 1 April 2008 (when the additional cardiac contraindications came into force) and 30 November 2009 (the cut-off date for available data in THIN). Although this analysis had several limitations, the percentage of patients being prescribed rosiglitazone despite contraindications being present (patients with cardiac failure or history of cardiac failure and acute coronary syndrome) was estimated to be around $8 \%$, indicating usage outside the approved label.

The EMA has closely monitored the cardiovascular issues with rosiglitazone over time, has exchanged views and information with other regulatory agencies worldwide, and has also twice consulted its Scientific Advisory Groups (SAGs) on Diabetes/Endocrinology and on Cardiovascular Issues on several questions related to TZDs and rosiglitazone in particular. In its meeting on 15 September 2008 the SAGs felt that the presumption of the increased cardiovascular risk associated with rosiglitazone had neither been confirmed nor fully refuted by the data available at that point in time. When asked about the appropriate place in therapy, the position was that rosiglitazone retained a small, though diminishing, place in therapy. On similar questions, the SAGs concluded on 17 July 2010 that, with regard to cardiovascular safety, the balance had shifted further against rosiglitazone and that a unique benefit over pioglitazone could not be identified. Therefore, no welldefined subgroup of patients more suitable for rosiglitazone than pioglitazone could be identified. The EMA's CHMP considered the possibility of further restrictions of the label as well as other additional risk minimisation measures, but did not consider them to be a feasible option in light of the difficulties in identifying such a subgroup.

Taking into account all available evidence, which has accumulated over time, on 22 September 2010 the EMA's CHMP recommended the suspension of the marketing of Avandia in the EU as the benefits no longer outweighed the risks. 


\section{Future role of cardiovascular outcome trials in the approval of drugs for diabetes}

The possibility of an increased cardiovascular risk associated with the use of rosiglitazone, but to some degree also the lack of evidence of a benefit with regard to macrovascular complications for glucose-lowering agents in patients with type 2 diabetes, raised questions from the medical community about the way medicines for the treatment of type 2 diabetes are approved [5-10]. This included criticism of the predominant reliance on $\mathrm{HbA}_{1 \mathrm{c}}$ as the primary outcome measure and an emphasis on the need for outcome studies. These issues triggered a broader review by the EMA of the guidance provided to industry on the development of diabetes drugs [20]. As a consequence, new draft guidance has been released for public consultation during the first part of 2010 and has attracted intensive feedback from stakeholders, including academia, such as from the EASD. Among other changes, the guideline will increase the data requirements for the clinical trials of new products to include a substantial population with a high cardiovascular risk and to further strengthen available safety data in general.

In a recent editorial, Lehman et al. raise important fundamental questions about the approval of new drugs for the treatment of diabetes [21], demanding better proof of long-term benefit and specifically requesting not only proof of long-term cardiovascular safety but also of long-term cardiovascular benefit. However, demonstration of the latter is unlikely to be a requirement anytime soon, as is evident from current regulatory draft guidelines on diabetes both from the EMA [22] and the FDA [23]:

Approval is currently based on $\mathrm{HbA}_{1 \mathrm{c}}$ as the primary outcome measure, in conjunction with a favourable risk profile, taking into account other potential benefits, such as effects on body weight, blood pressure and lipid profile. $\mathrm{HbA}_{1 \mathrm{c}}$ is a very well validated marker of glycaemic control and glucose control in itself is a therapeutic goal to treat the symptoms of hyperglycaemia. Moreover, a correlation between glycaemic control and a benefit with regard to microvascular complications (diabetic nephropathy, neuropathy and retinopathy) has been well established in type 2 diabetes [24]. However, the same degree of evidence does not exist for macrovascular complications. In fact, there is little unequivocal evidence of proven benefit with regard to macrovascular events for any diabetes drug, and as a result of this, none of the currently approved drugs for the treatment of type 2 diabetes via the EMA's centralised procedure has a claim for macrovascular benefit in its label. This, however, may change in the future. The increased requirements with regard to cardiovascular safety data $[22,25]$ will result in more cardiovascular outcome studies being performed. While in the first instance these may serve the purpose of demonstrating non-inferiority with regard to cardiovascular safety when compared with existing therapies, those trials may in some cases eventually demonstrate superiority, thus leading to a demonstrable benefit with regard to macrovascular events.

\section{Conclusion}

Rosiglitazone and its benefits and risks have stirred an intense debate in the medical community with sometimes rather divergent views. In the case of rosiglitazone, on 22 September 2010 the EMA's CHMP recommended the suspension of the product in the EU after taking into account all the available evidence which had accumulated over time, as well as the advice of its Scientific Advisory Groups. This included information from recent observational studies and meta-analyses, which heightened concerns regarding the cardiovascular risk of rosiglitazone, as well as the results of randomised controlled long-term studies. Despite a wide array of data, the evidence for a cardiovascular risk with rosiglitazone was not unequivocal, sometimes conflicting, but ultimately could not be refuted, and led EMA's CHMP to conclude that the benefits of rosiglitazone no longer outweigh the risks. In an era of increasing numbers of meta-analyses and observational studies, potential safety signals are more frequently emerging, whereas pitfalls of those analyses are not always immediately apparent.

As one of the consequences the EMA has already proposed draft guidance for data requirements concerning the knowledge of the safety profile in general, but also in particular with regard to cardiovascular events, of new drugs for the treatment of diabetes.

The EMA is committed to transparency of its decisions, and, while not releasing interim assessments of ongoing procedures, will make available a full detailed report (European Public Assessment Report) of the review of Avandia as soon as a European Commission Decision is finally issued.

Duality of interest The authors declare that there is no duality of interest associated with this manuscript.

\section{References}

1. European Medicines Agency recommends suspension of Avandia, Avandamet and Avaglim. Press release, 23 September 2010. Available from www.ema.europa.eu/docs/en GB/document library/Press_release/2010/09/WC500096996.pdf 
2. Cohen D (2010) Rosiglitazone: what went wrong? BMJ 341:c4848

3. EMEA (2000) European Public Assessment Report (EPAR) initial marketing authorisation documents-Avandia-Scientific Discussion. Available from www.ema.europa.eu/docs/en_GB/document library/EPAR_-_Scientific_Discussion/human/000268/WC 5 00029103.pdf [containing amendments up to 2004]

4. Nissen SE, Wolski K (2007) Effect of rosiglitazone on the risk of myocardial infarction and death from cardiovascular causes. N Engl J Med 356:2457-2471

5. Drazen JM, Morrissey S, Curfman GD (2007) Rosiglitazonecontinued uncertainty about safety. N Engl J Med 357:63-64

6. Montori VM, Gandhi GY, Guyatt GH (2007) Patient-important outcomes in diabetes - time for consensus. Lancet 370:1104-1106

7. Nathan DM (2007) Rosiglitazone and cardiotoxicity - weighing the evidence. N Engl J Med 357:64-66

8. Psaty BM, Furberg CD (2007) The record on rosiglitazone and the risk of myocardial infarction. N Engl J Med 357:67-69

9. Psaty BM, Furberg CD (2007) Rosiglitazone and cardiovascular risk. N Engl J Med 356:2522-2524

10. Rosen CJ (2007) The rosiglitazone story-lessons from an FDA Advisory Committee meeting. N Engl J Med 357:844-846

11. Home PD, Jones NP, Pocock SJ et al (2007) Rosiglitazone RECORD study: glucose control outcomes at 18 months. Diabet Med 24:626-634

12. Home PD, Pocock SJ, Beck-Nielsen H et al (2009) Rosiglitazone evaluated for cardiovascular outcomes in oral agent combination therapy for type 2 diabetes (RECORD): a multicentre, randomised, open-label trial. Lancet 373:2125-2135

13. Dormandy JA, Charbonnel B, Eckland DJ et al (2005) Secondary prevention of macrovascular events in patients with type 2 diabetes in the PROactive Study (PROspective pioglitAzone Clinical Trial In macroVascular Events): a randomised controlled trial. Lancet 366:1279-1289

14. Nathan DM, Buse JB, Davidson MB et al (2009) Medical management of hyperglycaemia in type 2 diabetes mellitus: a consensus algorithm for the initiation and adjustment of therapy: a consensus statement from the American Diabetes Association and the European Association for the Study of Diabetes. Diabetologia 52:17-30

15. Graham DJ, Ouellet-Hellstrom R, MaCurdy TE et al (2010) Risk of acute myocardial infarction, stroke, heart failure, and death in elderly Medicare patients treated with rosiglitazone or pioglitazone. JAMA 304:411-418
16. Nissen SE, Wolski K (2010) Rosiglitazone revisited: an updated meta-analysis of risk for myocardial infarction and cardiovascular mortality. Arch Intern Med 170:1191-1201

17. McEvoy B. 2010. Pioglitazone and rosiglitazone cardiovascular safety meta-analyses. FDA Advisory Committee, 13-14 July 2010. Available from www.fda.gov/downloads/AdvisoryCommit tees/CommitteesMeetingMaterials/Drugs/EndocrinologicandMeta bolicDrugsAdvisoryCommittee/UCM224739.pdf

18. Wertz DA, Chang CL, Sarawate CA, Willey VJ, Cziraky MJ, Bohn RL (2010) Risk of cardiovascular events and all-cause mortality in patients treated with thiazolidinediones in a managedcare population. Circ Cardiovasc Qual Outcomes 3:538-545

19. EMA (2010) THIN database study on rosiglitazone use. Available from www.encepp.eu/encepp/viewResource.htm?id=1592, accessed 7 October 2010

20. EMA (2008) Concept paper on the need for revision of the note for guidance on clinical investigation of medicinal products in the treatment of diabetes mellitus. Available from www.ema.europa. eu/docs/en_GB/document_library/Scientific_guideline/2009/09/ WC500003182.pdf

21. Lehman R, Yudkin JS, Krumholz H (2010) Licensing drugs for diabetes. BMJ 341:c4805

22. EMA (2010) Guideline on clinical investigation of medicinal products in the treatment of diabetes mellitus - draft. Available from www.ema.europa.eu/docs/en_GB/document_library/Scienti fic_guideline/2010/02/WC50007357̄0.pdf

23. US Department of Health and Human Services Food and Drug Administration Center for Drug Evaluation and Research (2008) Guidance for Industry. Diabetes Mellitus: Developing Drugs and Therapeutic Biologics for Treatment and Prevention-draft. Available from www.fda.gov/downloads/Drugs/GuidanceComplian ceRegulatoryInformation/Guidances/ucm071624.pdf

24. UK Prospective Diabetes Study (UKPDS) Group (1998) Intensive blood-glucose control with sulphonylureas or insulin compared with conventional treatment and risk of complications in patients with type 2 diabetes (UKPDS 33). Lancet 352:837-853

25. US Department of Health and Human Services Food and Drug Administration Center for Drug Evaluation and Research (2008) Guidance for Industry. Diabetes mellitus - evaluating cardiovascular risk in new antidiabetic therapies to treat type 2 diabetes. Available from www.fda.gov/downloads/Drugs/GuidanceComplian ceRegulatoryInformation/Guidances/ucm071627.pdf 